\title{
Intermittent Weakness and Mediastinal Weakening
}

\author{
Umer Feroze Malik*, Mersadies R. Martin, Hien D. Pham, \\ Ahmed Mahmoud, Sheela Kapre
}

\section{INTRODUCTION}

The association of myasthenia gravis (MG) with thymoma is about $15 \%$, which increases to about $35 \%$ in older patients $(1,19)$. Thymomas represent nearly $50 \%$ of tumors in the anterior mediastinum and if large enough may cause mediastinal widening (12). The connection between the thymus gland and MG has led to the medical recommendation of a thymectomy, however, a thymectomy does not cure MG but may significantly decrease the symptoms in $90 \%$ of patients with MG (13). If a female between the ages of 20-30 or a male older than 60 complains of generalized fatigue, eye muscle weakness, dysphagia, dystonia, and/or dysarthria MG should be considered and thymoma ruled out $(1,3,4)$. This case report provides detailed descriptions and illustrations of how to recognize and treat $\mathrm{MG}$, the importance of investigating for a thymoma, and current information regarding a thymectomy. Overall, even though evidence has shown the correlation between MG with thymoma, this case report depicts how a patient may present with multiple complaints yet specific to the diagnoses illustrating the importance of conducting a thorough history and physical.

\section{CASE PRESENTATION}

A 57-year-old Caucasian male with no known past medical history came into the Emergency Department complaining of progressively worsening dizziness, jaw weakness, difficulty swallowing, and left eyelid drooping for one-to-two weeks. Also, the patient stated that he was unable to continuously chew his food and

\footnotetext{
*To whom correspondence should be addressed:

Umer Feroze Malik, MD

P.O Box 1020

San Joaquin General Hospital

500 West Hospital Road

French Camp, Ca

95231

umalik@sjgh.org
}

had to take breaks in order to finish his meals. In addition, he complained of sporadic episodes of blurriness and double vision for at least three months. He denied trauma, falling and/or febrile illnesses prior to his current symptoms. Upon further questioning, the patient stated that his voice had recently changed to a nasal tone. He did not seek medical attention earlier in hopes that his symptoms would spontaneously resolve.

Past medical history was unremarkable. Past surgical history included a tonsillectomy at the age of 12 , bilateral reconstructive mastoid surgery at the age of 16 with a revision surgery shortly after for left facial paralysis caused by a compressed facial nerve.

Family history included his mother who had diabetes and passed away from end stage renal disease. The patient's father died at the age of 91 with no known morbidities. His brother and sister both suffer from diabetes and hypertension. There are no known cancers or neurological disorders within his family.

The patient used to work in construction and frequently used a jackhammer. He currently works at a flea market moving and lifting heavy merchandise. He lives alone and has been separated from his wife for three months. He has three healthy children. He has no history of cigarette smoking or intravenous drug abuse and occasionally drinks alcohol within a social atmosphere. He reports no history of recent travel, camping or hiking. The patient was not taking any medications. His only allergy was penicillin that resulted in a rash.

Review of symptoms included intermittent occurrences of difficulty speaking, chewing and swallowing, slowed thinking process, and a constant nasal tone in his voice for one-to-two weeks. Additionally, the patient complained of double vision and left sided earache for three months. When asked about the current symptoms the patient gave inconsistent information and said that he feels fatigued most of the time and his symptoms got worse as the day 
progressed but improved with rest. Prior to this current illness his appetite was fine. He further denied weight changes, shortness of breath, chest pain, back pain, bowel or bladder incontinence, and skin rashes.

Physical examination revealed a middle-aged moderately built and well-nourished Caucasian male in no apparent distress with bilateral ptosis. Vital signs were normal. Pupils were equal but slowly reactive to light. The patient was unable to look upwards and noted to have bilateral vertical gaze palsy. When directed to move his eyes lateral, the right could move more than the left yet they were both delayed. The rest of the Head and Neck examination was normal. Face examination showed a slightly less prominent left nasolabial fold compared to the right, and bilateral facial muscle weakness was appreciated when frowning or smiling. There was no tongue or uvula deviation found. The cardiovascular, pulmonary, abdominal examination, extremities, rectal and prostate exam was normal. The neurological examination revealed a patient that was alert and oriented to person, place and time. In all extremities, the motor examination was $4+/ 5$ and deep tendon reflexes were $2+$. The patient had a strong gag reflex. As noted above, cranial nerves two, three, five, six, and seven were affected. The patients gait was intact, no instability noted, and the cerebellum and its reflexes were intact.

The differential diagnoses in this case were $\mathrm{MG}$, botulism, multiple sclerosis and/or a brain stem infarct. An important feature to differentiate $\mathrm{MG}$ from botulism is upon examination of pupils. In MG accommodation of the pupils is usually spared, whereas botulism presents with impaired accommodation and gastrointestinal symptoms simultaneously (21.). Multiple sclerosis and brain stem infarct requires objective evidence of neurologic signs that are localized to the brain or spinal cord which were absent in our patient.

The laboratory values regarding a complete blood count, basic metabolic panel, liver function test, thyroid function test, lipoproteins, triglycerides, urine analysis, and urine culture were all within normal limits.

In regards to the patients' symptoms, he was admitted with a diagnosis of weakness secondary to possible myasthenia gravis. His treatment began with oral Prednisone and oral pyridostigmine. A medical nutritional therapy consult recommended a modified mechanical soft diet secondary to complaints of dysphagia.

On this same day of admission, a lumbar puncture was done with a cerebral spinal fluid (CSF) cell count, differential, culture, protein and glucose amount measured to be within normal limits with exception of the CSF having a high red blood cell count of 34 cells/centimeter ${ }^{3}$ (normal is zero). In addition, the CSF was negative for oligoclonal bands. These CSF result made the diagnosis of CNS disorders such as the Guillain-Barre Syndrome and Multiple Sclerosis less likely. Chest x-ray showed anterior mediastinal widening with left basilar subsegmental atelectasis. There was also a mild elevation of the left hemidiaphragm and minor degenerative changes of the thoracic spine. Computed tomography (CT) without contrast of the head showed no acute intracranial abnormalities. The CT impression concluded an anterior mediastinal mass measuring approximately seven centimeters $(\mathrm{cm})$ in length by four $\mathrm{cm}$ in diameter with irregular calcifications (Figure 1). However, malignancy was neither ruled in nor ruled out. At this point, the likely diagnosis was a thymoma.

Nerve conduction studies and electromyography were completed which revealed findings most consistent with a postsynaptic neuromuscular junction transmission disorder. The acetylcholine receptor antibodies were positively elevated at 116.3 nanomoles/liter $(\mathrm{nmol} / \mathrm{L})$ (reference range $<0.1$ ). At this point, myasthenia gravis was the confirmed diagnosis and the plan was to remove the thymus gland and keep the patient on Prednisone and pyridostigmine throughout the hospital stay.

On the fifth day of admission, the patient underwent an uncomplicated thymectomy. The surgeons used a median sternotomy approach to dissect the thymus gland. Pathology reported both gross and microscopic descriptions of the isolated thymus. The findings were consistent with the World Health Organization (WHO) type B3 with involvement of minimal invasion. The neoplasm was not present at the surgical resection margins.

During the remainder of the hospital course Prednisone was continuously tapered down to prevent wound dehiscence, a five-day course of intravenous immunoglobulins was administered. Furthermore, there were marked improvements noted in the tone of the facial muscles bilaterally. Significant improvements in the symptoms of weakness and dysphagia were also found.

On postoperative Day 10, an acetylcholine receptor binding test showed a significant decrease compared to the preoperative measurement $(116.3 \mathrm{nmol} / \mathrm{L}$ and 33.3 $\mathrm{nmol} / \mathrm{L}$, respectively). The patient was discharged on postoperative day 13 with pyridostigmine, a tapering dose of Prednisone, and vitamin A for 14 days.

Follow-ups two years postoperatively have shown significant improvements on clinical examination. The patient has been monitored closely on low dose steroids and pyridostigmine and has only reported minor symptoms of MG. 


\section{DISCUSSION}

Myasthenia gravis (MG) is an autoimmune disorder and neuromuscular disease with characteristic symptoms of unpredictable and irregular bouts of muscle weakness and fatigability (1). MG is mediated by circulating anti-acetylcholine receptor antibodies that block acetylcholine receptors at the postsynaptic neuromuscular junction (2). A characteristic finding associated with MG is a bimodal peak incidence that usually occurs in females between the ages of 20 to 30 years and males greater than 60 years (3). The onset of this disorder is often sudden with reports of intermittent symptoms that become worse during periods of activity and improve after periods of rest (1). Feature signs and symptoms include weakness in the muscles that control eye and eyelid movements (ocular myasthenia), facial expression, chewing, swallowing (dysphagia), slurred speech (dysarthria) that is often associated with a nasal tone (dystonia) due to weakness of the velar muscles, and generalized muscle weakness that may involve the extremities and trunk (4). Ocular myasthenia symptoms vary in severity but usually include asymmetrical drooping of one or both eyelids (ptosis) and double vision (diplopia). In fact it is these two symptoms that are usually first reported by patients with MG (5). It is also worth mentioning that the occurrence of dysphonia and dysphagia are found to be the first symptoms related to late-onset MG (6). In severe cases, the muscles that control respiration can be affected (myasthenia crisis), and when this happens assisted ventilation is often required to sustain life $(6,20)$. In patients whose respiratory muscles are already weak crisis may be triggered by infection, fever, or by an adverse reaction to a medication (6).

Diagnosis of MG is based on a thorough physical examination, clinical testing, serologic testing, and imaging studies to rule out the commonly associated thymoma. In addition to the signs and symptoms as described above, other examinations that may facilitate the diagnosis of MG include applying ice to fatigued muscles to observe a characteristic increase in strength, and by performing and monitoring the following tests to observe the muscle fatigability (8).

Clinical testing may involve the Edrophonium test, electromyography, and/or in severe cases, pulmonary function tests. The Edrophonium test is valuable when the diagnosis of MG is unconfirmed. This test is positive if there is increased muscle strength (especially observed in the eyes) after the administration of intravenous edrophonium chloride or neostigmine, drugs that temporarily increase the amount of acetycholine at the neuromuscular junction (9). Side effects associated with this test are usually mild (such as urinary or fecal incontinence and excessive salivation). Severe bradycardia and bronchoconstiction are rare risks associated with this test but can happen if parasympathetic supply of the cardiopulmonary system becomes over active. It is because of these effects closer cardiac monitoring is advised while performing this test. Also Edrophonium administration should be performed in a setting where immediate ventilatory support can be provided if needed. On the other hand, electromyography is considered to be one of the most sensitive tests in diagnosis of MG. Electromyography measures muscle fatigability by stimulating repetitive electrical impulses to muscle fibers (8).

Serologic testing is effective in testing the blood for antibodies against the acetylcholine receptor. It has been found that approximately $80 \%$ of people with MG have increased serum antibodies to the acetylcholine receptor and the remaining $20 \%$ have antibodies to the muscle specific kinase (10).

Imaging studies are usually taken in patients with $\mathrm{MG}$ because studies have found the association of MG with thymoma to be about $15 \%$, and $35 \%$ in older patients (Brachmann K, 1). A thymoma is considered a rare tumor of the thymus gland that is usually benign but when it becomes malignant it is extremely invasive (11). Thymomas represent about $50 \%$ of tumors in the anterior mediastinum and may grow in size and eventually squeeze blood vessels, the heart and/or lungs (12). The connection between the thymus gland and MG has led to the medical recommendation of a thymectomy. However, a thymectomy does not cure MG but may significantly decrease the symptoms of MG in $90 \%$ of patients (13).

The value of thymectomy is dependent on a high proportion of B-lymphocytes and the germinal center B-lymphocytes in the thymus, suggesting that one of the biological roles of thymectomy in the treatment of MG is removing the thymus-associated germinal centers (14). Although the thymus is the central organ for Tlymphocyte differentiation, the germinal center is where

\begin{tabular}{|l|l|}
\hline Signs & Description \\
\hline Shifting Ptosis & $\begin{array}{l}\text { Quickly fluctuating droop of the eye } \\
\text { lids which is usually asymmetric. }\end{array}$ \\
\hline Curtain Sign & $\begin{array}{l}\text { Ptosis is enhanced if the patient } \\
\text { keeps one eye open by holding it, } \\
\text { this leads the other eye to droops } \\
\text { more ( like a curtain) }\end{array}$ \\
\hline $\begin{array}{l}\text { Lid Twitch } \\
\text { sign }\end{array}$ & $\begin{array}{l}\text { Eye lid twitch for fraction of a } \\
\text { second before settling when the eyes } \\
\text { are opened after gentle closure. }\end{array}$ \\
\hline Peek sign & $\begin{array}{l}\text { Upon tight closure of the eyes, the } \\
\text { lid margins start to separate to the } \\
\text { point that white of the sclera } \\
\text { becomes visible. }\end{array}$ \\
\hline
\end{tabular}

Table 1: Specific sigs to elicit muscle fatigability 
where B-lymphocytes differentiate to the cells producing antibodies (i.e. acetylcholine receptor antibodies) with high affinity to the antigens. Consistent with the improvement of clinical symptoms, the anti-acetylcholine receptor antibody was shown to decrease following thymectomy (10). With that in mind, an anti-acetylcholine receptor antibody may be considered a good postoperative indicator of improvement. In this case, all the symptoms significantly decreased postoperatively as did the antiacetylcholine receptor antibodies from 116 to 33.3 nmols/L.

In the new WHO classification, the types of thymoma are distinguished on histological criteria which seem to be of independent prognostic significance: 1) type A thymomas (medullary or spindle-cell); 2) type $A B$ thymomas (mixed thymoma); 3) type B thymomas with subclasses B1 (lymphocyte-rich), B2 (cortical) and B3 (epithelial, atypical, well-differentiated); 4) type $\mathrm{C}$ (thymic carcinomas). Type $\mathrm{A}$ and $\mathrm{AB}$ are usually benign, types B1-B3 is considered low to moderate malignant neoplasms, and type $\mathrm{C}$ is usually highly malignant (15). Interestingly and not well analyzed was a study that found type B3 thymomas, as presented in this case, to show chromosomal imbalances with a gain of 1q, loss of chromosome 6 , and loss a of 13q (16). The most recent WHO classification of thymoma has acknowledged that surgical resection remains the mainstay of treatment for type B3 thymic tumors, and radiation and chemotherapy also have been applied widely as adjuvant and palliative procedures (17).

Thymectomies can be performed using several surgical approaches. Ranging from most to least invasive is the full median sternotomy, partial sternotomy, thoracoscopic, and transcervical approaches (18). In this case, the full median sternotomy approach was used and an incision was made down the middle of the chest through the sternum in order to expose the enlarged thymoma. This technique was chosen because CT of the chest showed a massive widening of the anterior mediastinum, and due to the size of the thymoma an invasive approach was necessary.

The long-term results of patients who underwent a thymectomy for MG was recently studied paying special attention to postoperative disease-related outcome, quality of life, and differences regarding the operative approach. Overall, this study concluded that the transsternal thymectomy contributed to an improvement in MG symptoms for all subgroups, and minimally invasive surgery was found to be superior in terms of improvement in MG-associated symptoms (19). Although it has been shown that removal of thymoma can be beneficial in MG, It is not clear if a hyperplastic or normal thymic tissue should be removed as some authors have concluded exacerbation of $\mathrm{MG}$ with removal of hyperplastic or normal thymic tissue. The details of this controversy are beyond the scope of this article. Some authors have also shown that even though thymoma removal has minimal effects on the symptoms progression, it should still be removed primarily for oncological purposes.

Thymectomy is only one component of the treatment for MG. Medical therapy of MG may also include anticholinesterase inhibitors, such as pyridostigmine, and/or immunosuppressive medications, such as corticosteroids, plasmaphoresis or intravenous immunoglobulin (20). Postoperative worsening of MG in this case was probably secondary to the thymectomy and the patient having preexisting mild atelectasis, and most likely not due to an excess of cholinergic medications. However, cholinesterase inhibitors should be stopped when myasthenia crisis (respiratory and pharyngeal muscle paralysis) is suspected because it may be difficult to distinguish between myasthenia crisis versus cholinergic crisis. Regardless, patients may require prolonged mechanical ventilatory support, and as seen in this case, elective intubation following surgery. Alternatively, medications known to worsen MG symptoms include D-penicillamine, neuromuscular blocking agents, quinidine, aminoglycoside antibiotics, beta-blockers, calcium channel blockers, magnesium salts, and iodinated contrast agents (20). Therefore, a careful review of a patient's medication regime with other co-morbidities is imperative.

\section{CONCLUSION}

When the signs and symptoms of MG are suspected and imaging studies reveal widening of the anterior mediastinum it is important to know that a thymoma may represent about $50 \%$ of tumors in the anterior mediastinum (12). A thymectomy may not cure MG but may significantly decrease the symptoms (13), and further prevent the rapid conversion of a benign tumor into a highly invasive tumor (11). Testing the blood for antibodies against the acetylcholine receptor before and after a thymectomy may assist in the diagnosis and treatment of patients with MG (10). The prognosis of a patient with MG who undergo a thymectomy has not been studied thoroughly as there are some discrepancies regarding remission and recurrence rates, however having a thymectomy has shown a better outcome than not having one (19).

\section{REFERENCES}

1. Drachman DB. Myasthenia gravis. $N$ Engl J Med 1994;330:1797-810. 
2. Conti-Fine BM, Milani M, Kaminski HJ. Myasthenia gravis: past, present, and future. J. Clin. Invest. 2006;116 (11): 2843-54.

3. Slesak G, Melms A, Gerneth F, et al. Late-onset myasthenia gravis: follow-up of 113 patients diagnosed after age 60. Ann NY Acad Sci 1998;841:777-80.

4. Rastenyte D, Vaitkus A, Neverauskas R, Pauza V. Demographic characteristics of patients with myasthenia gravis. Medicina 2002;38(6):611-6.

5. Thomas S, Critchley P, Lawden M et al. Stiff person syndrome with eye movement abnormality, myasthenia gravis, and thymoma. Journal of Neurology, Neurosurgery \& Psychiatry 2005;76(1):141-142.

6. Chua E, McLoughlin C, Sharma A. Myasthenia gravis and recurrent falls in an elderly patient. Age Ageing 2000;29:83-4.

7. Bedlack RS, Sanders DB. How to handle myasthenic crisis. Essential steps in patient care. Postgrad Med 2000;107 (4): 211-4, 220-2.

8. Scherer K, Bedlack RS, Simel DL. Does this patient have myasthenia gravis? JAMA 2005;293 (15): 1906-14.

9. Pascuzzi RM. The edrophonium test. Semin Neurol 2003;23:83-8.

10. Leite MI, Jacob S., Viegas S., et al. IgG1 antibodies to acetylcholine receptors in 'seronegative' myasthenia gravis. Brain 2008;131(7):1940-1952.

11. Pan CC, Chen CC, Wang LS et al. Thymoma is associated with an increased risk of second malignancy. Cancer 2001; 92: 2406-2411.

12. Welsh JS, Wilkins KB, Green R et al. Association between thymoma and second neoplasms. JAMA 2000; 283: $1142-1143$.
13. Mussi A, Lucchi M, Murri L, et al. Extended thymectomy in myasthenia gravis: a team-work of neurologist, thoracic surgeon and anaesthesist may improve the outcome. European Journal of Cardio-Thoracic Surgery 2001;19(5):570-575.

14. Okumura M, Ohta M, Takeuchi Y. The immunologic role of thymectomy in the treatment of myasthenia gravis: implication of thymus-associated B-lymphocyte subset in reduction of the anti-acetylcholine receptor antibody titer. J Thoracic Cardiovasc Surg 2003;126:1922-1928.

15. Marx A, Muller-Hermelink HK. Thymoma and thymic carcinoma. Am J Surg Pathol 1999;23:739-742.).

16. Zettl A, Strobel P, Wagner K. Recurrent Genetic Aberrations in Thymoma and Thymic Carcinoma. American Journal of Pathology 2000;157:257-266.).

17. Kondo K. Optimal therapy for thymoma. J. Med. Invest 2008;55:17-28.

18. Yim AP. Paradigm shift in surgical approaches to thymectomy. ANZ Journal of Surgery. 2002;72(1):40-45.

19. Bachmann K, Burkhardt D, Schreiter I, et al. Long-term outcome and quality of life after open and thoracoscopic thymectomy for myasthenia gravis: analysis of 131 patients. Surg Endosc 2008;22(11):2470-7.

20. Kanzaki M, Obara T, Sasano S. Long-Term Clinical Outcome after Extended Thymectomy Combined Postoperative HighDose Steroid Therapy for Juvenile Myasthenia Gravis. Ann. Thorac. Cardiovasc. Surg. 2008;14(2):119-122.

21. Penas S, Faria O, Rosario S, et al. Opthalmic manifestations in 18 Patients with Botulism Diagnosed in Porto, Portugal Between 1998 and 2003. Journal of Neuro-Ophthalmology 2005;25(4):262-267.). 